\title{
Poststroke pain - but multiple pain mechanisms
}

\author{
Vinjamuri Chari MB BS MD FRCPC ${ }^{1}$, Eldon Tunks MD FRCPC ${ }^{2}$
}

V Chari, E Tunks. Poststroke pain - but multiple pain mechanisms. Pain Res Manage 2010;15(1):24-26.

A 42-year-old man presented with acute left hemiplegia due to a right frontotemporal hemorrhagic stroke and left-sided pain. While the initial presentation suggested central poststroke pain, subsequent investigations also implicated heterotopic ossification of the left hip and amplification of previous low back pain by the new central pain. While heterotopic ossification has been commonly associated with brain injury, spinal cord injury or osseous injury, it is only rarely associated with stroke.

Poststroke pain may be multifactorial, and discovering the pain mechanisms has important implications for treatment.

Key Words: Central poststroke pain; Heterotopic ossification; Stroke

\section{Une douleur aux nombreux mécanismes après un accident vasculaire cérébral}

Un homme de 42 ans a consulté à cause d'une hémiplégie gauche aiguë causée par un accident vasculaire cérébral (AVC) frontotemporal droit, ainsi que d'une douleur à gauche. Les manifestations initiales laissaient croire à une douleur centrale après un AVC, mais les explorations subséquentes démontraient également une ossification hétérotopique de la hanche gauche et une douleur lombaire antérieure, amplifiée par la nouvelle douleur centrale. L'ossification hétérotopique s'associe souvent à un traumatisme cérébral, médullaire ou osseux, mais rarement à un AVC. La douleur après un AVC peut être multifactorielle, et il est important de découvrir les mécanismes de la douleur pour déterminer un traitement.

In addition to antihypertensive medication and warfarin, his admission medication included gabapentin, codeine, baclofen and venlafaxine, all in therapeutic doses, but he was still complaining of pain. Nursing and physiotherapy staff noted that he was complaining of widespread burning pain in the left side, aversion to being touched, and that this pain was interfering with function and rehabilitation. Even attempts to reposition his left leg evoked aversion and pain complaint.

Mental status and standardized testing revealed adjustment disorder characterized by anxiety and depressed mood, as well as a propensity to substance abuse. He reported poor sleep quality. He had a Mini-Mental State Examination score of 30 (maximum score of 30) and a Montreal Cognitive Assessment score of 24 (maximum score of 30).

A physical examination elicited a complaint of pain, with a reduced passive range of motion affecting the left shoulder, and left hip pain with external rotation of the left leg. There was tenderness in the periscapular, neck and left shoulder areas.

He exhibited no aphasia or dysarthria, and had homonymous left inferior quadrantanopia with mild left central facial paresis and dense left hemiplegia.

There was reduction in simple (single) touch sensation in the whole left body side compared with the right, but he could not tolerate tapping touch, brushing or sustained pressure on the left upper and lower body side. He reported pain on light brushing or finger tapping, particularly around the left groin, and to application of a cold pack on the upper or lower left body side. Hyperalgesia to pin-scratch and pin-prick was noted in the left groin, arm and leg.

${ }^{1}$ Division of Physical Medicine and Rehabilitation; ${ }^{2}$ Department of Psychiatry, McMaster University, Regional Rehabilitation Centre, Hamilton Health Sciences, Hamilton, Ontario

Correspondence: Dr Vinjamuri Chari, Regional Rehabilitation Centre, 300 Wellington Street North, Hamilton, Ontario L8L 0 A4.

Telephone 905-521-2100 ext 40815, fax 905-577-8233, e-mail chari@hhsc.ca 


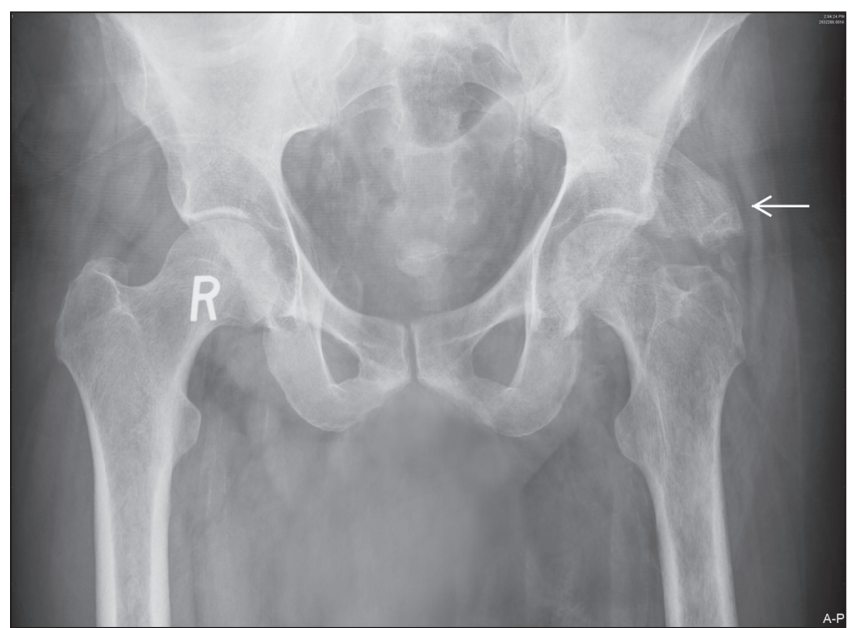

Figure 1) Anteroposterior radiological view of the pelvis performed on September 7, 2007, showing superolateral heterotopic ossification of the left hip joint (arrow)

The initial diagnostic impression was that a right cerebral hemorrhagic stroke had resulted in onset of central poststroke pain, which, in turn, may have distorted and amplified the preexisting mechanical back pain with pain referral to the hip and groin.

The initial pain management was a prescription of sustained-release morphine, and ratio-Oxycocet (oxycodone and acetaminophen, ratiopharm, Canada) for breakthrough pain, while codeine was discontinued. Gabapentin was changed to pregabalin. He was scheduled for rehabilitation with the physiotherapy and occupational therapy services. The pain in the left groin and hip was still a problem at this point.

Because of groin pain on external rotation of the hip, hip pathology was suspected. However, a left hip joint x-ray did not reveal arthropathy or derangement, and closer examination of the $\mathrm{x}$-ray showed what appeared to be heterotopic ossification (Figure 1). Erythrocyte sedimentation rate was $40 \mathrm{~mm} / \mathrm{h}$ with normal white blood cell count and alkaline phosphatase.

A triple-phase bone scan showed generalized increased uptake in the distal left arm, leg and left trochanter region, consistent with heterotopic ossification (Figure 2).

The radiologist's report queried whether the triple-phase bone scan was also consistent with complex regional pain syndrome (CRPS); as well, the patient complained of limitation and burning pain, and he had clinical signs of hyperalgesia. However, there were no symptoms or signs of vasomotor changes (temperature or skin colour asymmetry), sudomotor changes (hyperhydrosis), or swelling or dystrophic changes in the skin or appendages. The diagnostic criteria for CRPS were not clinically met.

If heterotopic ossification is present, pain and impairment should improve with nonsteroidal anti-inflammatory drug treatment. A three-month treatment with indomethacin was started for management of the heterotopic ossification, resulting in reduction in the hip pain, increased sitting tolerance and increased wheelchair mobility. Erythrocyte sedimentation rate, which was elevated on admission at $40 \mathrm{~mm} / \mathrm{h}$, fell within normal range a few weeks after initiating indomethacin.

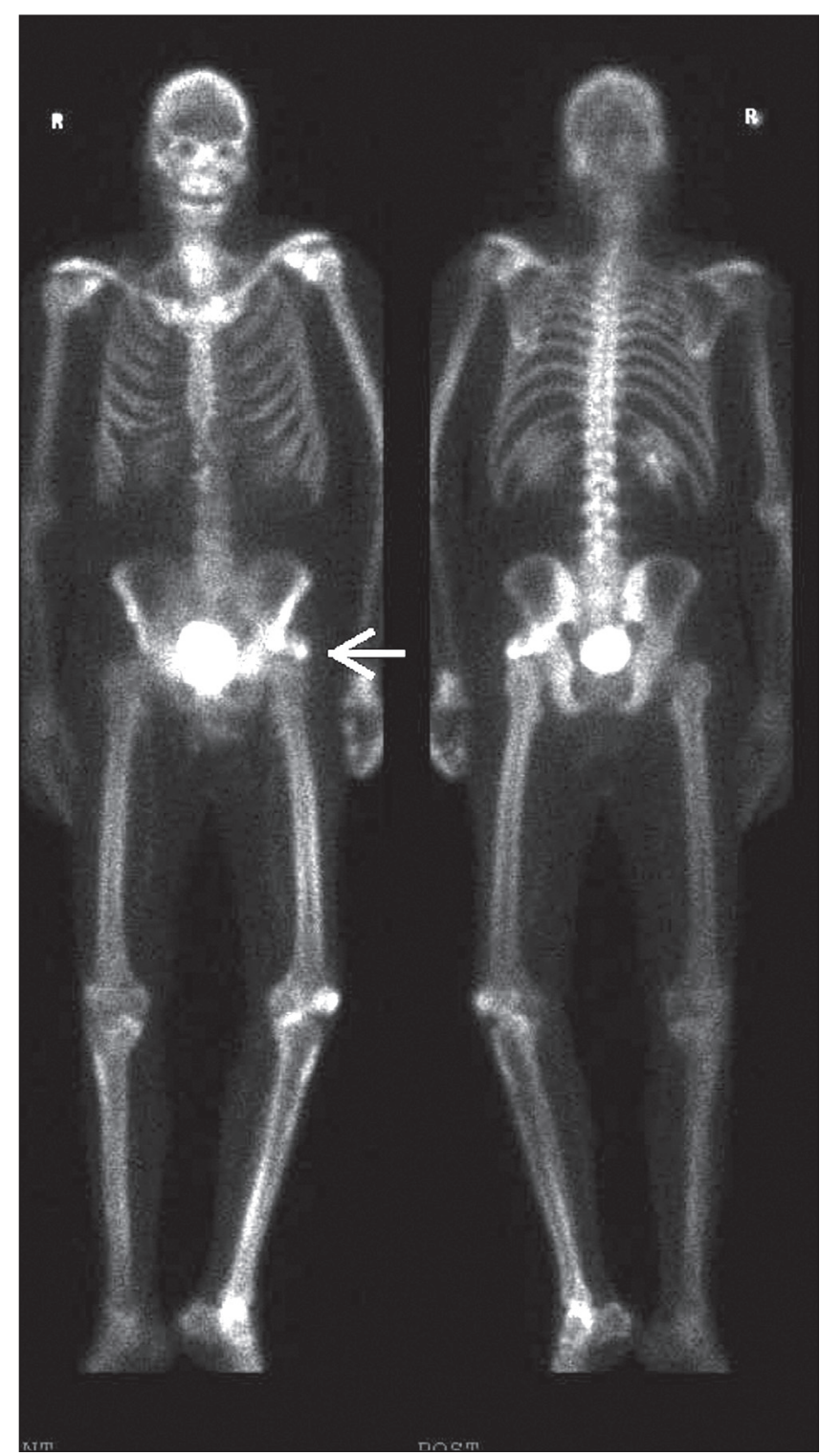

Figure 2) Triple-phase whole body scan performed September 19, 2007, showing mild diffuse increased uptake in the left arm and leg, and also increased uptake in the left hip superior to the greater trochanter (arrow), consistent with heterotopic ossification

\section{DISCUSSION}

The present case is useful in illustrating the variety of pain mechanisms that may be associated with poststroke status, and the value of assessing pain mechanisms at the bedside and with imaging to plan effective pain management. The pain mechanisms included previous mechanical low back pain, although the patient had been able to function and work; stroke followed by onset of probable central poststroke pain, which, in turn, probably amplified the pre-existing low back pain on the side of the stroke; and onset of heterotopic ossification that required nonsteroidal anti-inflammatory drug treatment.

While heterotopic ossification is commonly associated with injury to the brain or spinal cord, or osseous injury, it is only infrequently associated with stroke. A few reports in the North American literature estimate the incidence of this complication at $1 \%$ or less in the stroke population (3-5). 
CRPS was considered in the differential diagnosis, but clinical signs and symptoms did not make the threshold for that diagnosis. It is difficult to diagnose CRPS using only a bone scan.

Central poststroke pain, previously called 'thalamic syndrome', was considered likely. Features suggestive of central poststroke pain include reduced acuity to touch sensation in the whole left side, allodynia, hyperalgesia, and spontaneous burning pain in the left upper and lower body (including intolerance of repeated touch, pressure or cold stimuli in the whole left body side). Assuming that central poststroke pain may be present, and noting that the patient had a history of low back pain, amplification of pre-existing mechanical back pain by central pain is a possible explanation of his markedly increased pain in the left hip and groin after the stroke.

It is not clear whether central poststroke pain syndrome could correlate with increased diffuse uptake on bone scan, and the authors are not aware of any reports linking central poststroke pain with increased diffuse uptake on technetium bone scan. However, paresis of a limb may augment blood pooling and explain the image.

In the patient presenting with central poststroke pain, identified pain mechanisms included previous low back pain; onset of heterotopic ossification after stroke, as the dominant pain mechanism, complicated the pain affecting the left body side. Significant improvement occurred with treatment of the heterotopic ossification using indomethacin, along with physical therapy to restore function.

\section{REFERENCES}

1. Jönsson AC, Lindgren I, Hallström B, et al. Prevalence and intensity of pain after stroke: A population-based study focusing on patients' perspectives. J Neurol Neurosurg Psychiatry 2006;77:590-5.

2. Andersen G, Vestergaard K, Ingemann-Nielsen M, et al. Incidence of central post-stroke pain. Pain 1996;61:187-93.

3. McCarthy EF, Sundaram M. Heterotopic ossification: A review. Skeletal Radiol 2005;34:609-19.

4. Banovac K. Heterotopic ossification. <www.emedicine.com/pmr/ topic51.htm > (Accessed on February 1, 2010).

5. Hajek VE. Heterotopic ossification in hemiplegia following stroke. Arch Phys Med Rehabil 1987;68:313-4. 


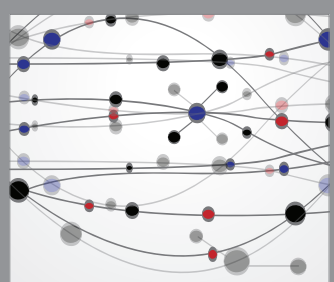

The Scientific World Journal
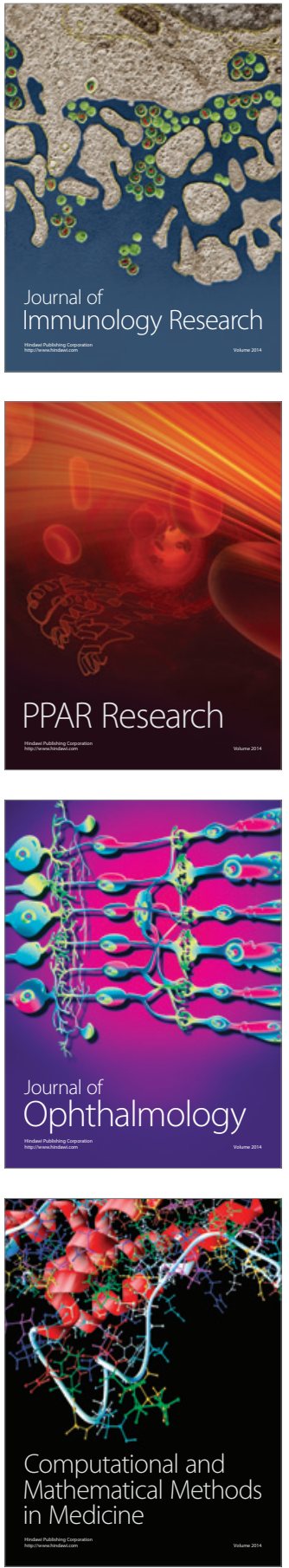

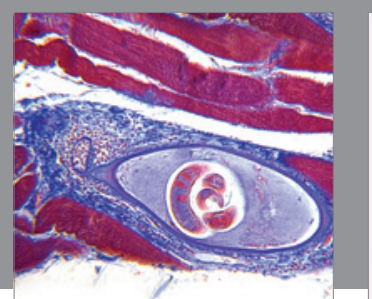

Gastroenterology Research and Practice

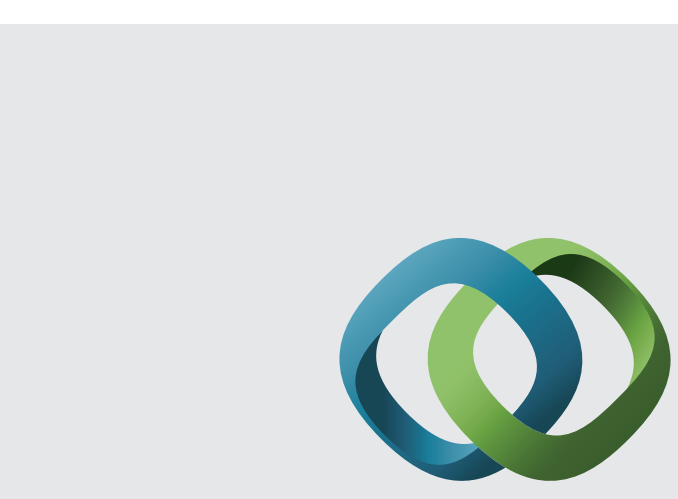

\section{Hindawi}

Submit your manuscripts at

http://www.hindawi.com
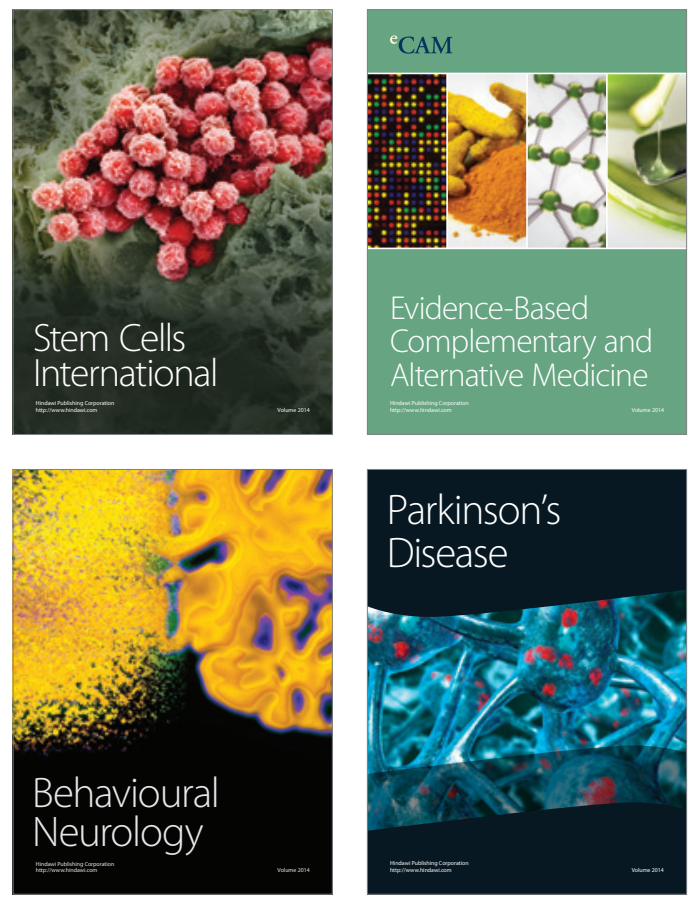
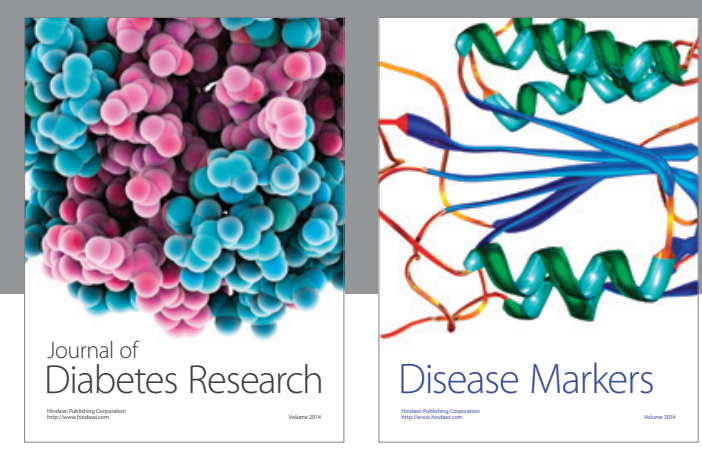

Disease Markers
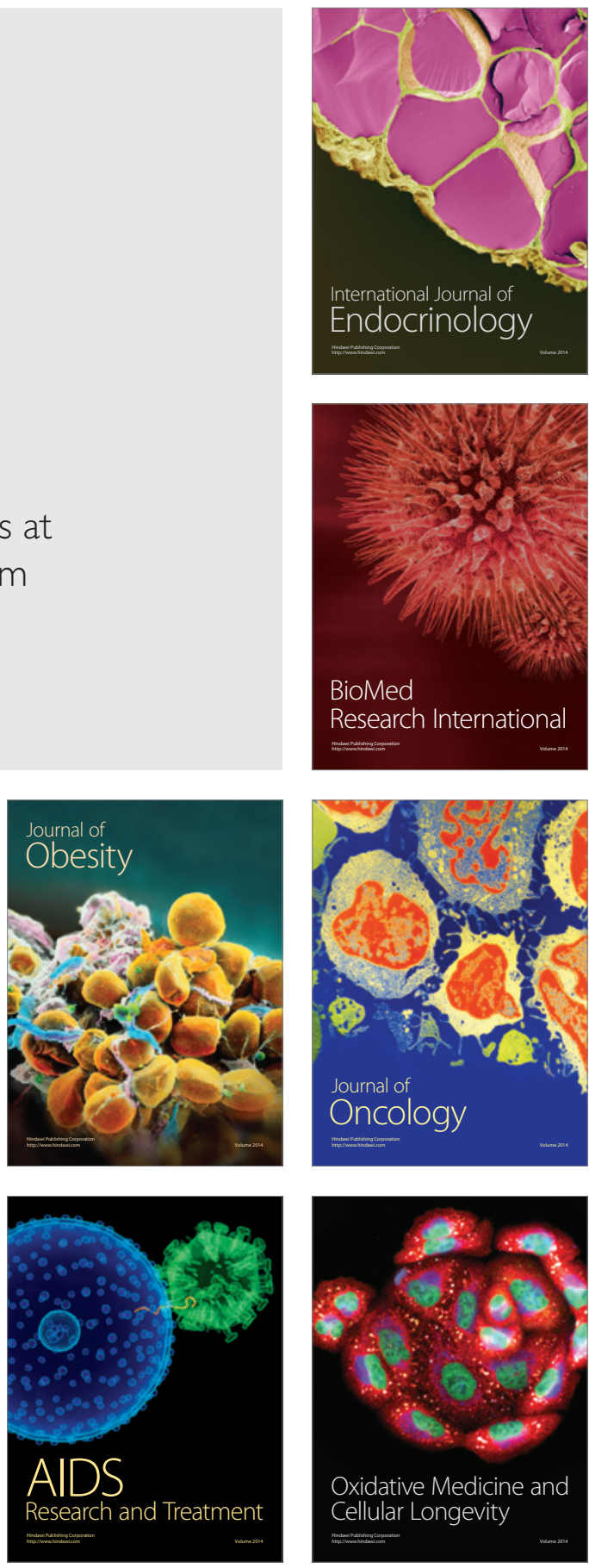